3. Viscusi DJ, King WP, Shaffer RE. Effect of decontamination on the filtration efficiency of two filtering facepiece respirator models. J Int Soc Respir Prot 2007;24:93-107.

4. Martin SB, Mayor ES. 33. Electrostatic respirator filter media: filter efficiency and most penetrating particle size effects. Appl Occup Environ Hyg 2000; 15:609-617.
5. Lindsley WG, Martin SB, Thewlis RE, et al. Effects of ultraviolet germicidal irradiation (UVGI) on N95 respirator filtration performance and structural integrity. J Occup Environ Hyg 2015;12:509-517.

6. Lindsley WG, Martin SB, Thewlis RE, et al. Evaluation of five decontamination methods for filtering facepiece respirators. Ann Occup Hyg 2009; 12:509-517.

\title{
Environmental cleaning is effective for the eradication of severe acute respiratory syndrome coronavirus 2 (SARS-CoV-2) virus in contaminated hospital rooms: A patient from the Diamond Princess cruise ship
}

\author{
Yosuke Hirotsu $\mathrm{PhD}^{1}$ (1), Makoto Maejima BS², Masumi Nakajima BN ${ }^{3}$, Hitoshi Mochizuki MD ${ }^{1,4}$ and Masao Omata MD ${ }^{4,5}$ \\ ${ }^{1}$ Genome Analysis Center, Yamanashi Central Hospital, Kofu, Yamanashi, Japan, ${ }^{2}$ Division of Microbiology in Clinical Laboratory, Yamanashi Central Hospital, \\ Kofu, Yamanashi, Japan, ${ }^{3}$ Division of Infection Disease Control, Yamanashi Central Hospital, Kofu, Yamanashi, Japan, ${ }^{4}$ Department of Gastroenterology, \\ Yamanashi Central Hospital, Kofu, Yamanashi, Japan and ${ }^{5}$ The University of Tokyo, Tokyo, Japan
}

To the Editor-Doctors, nurses, and other medical staff are greatly concerned about nosocomial outbreaks of severe acute respiratory syndrome coronavirus 2 (SARS-CoV-2). Environmental contamination is a possible source of nosocomial transmission., ${ }^{1,2}$ However, how effective environmental cleaning is against SARS-CoV-2 remains unclear.

A 75-year-old man infected with SARS-CoV-2 was diagnosed with COVID-19 during the quarantine period on the Diamond Princess cruise ship. He was transferred directly to our hospital on February 11, 2020. He resided in patient room A for 2 days then was moved to room B, where he stayed for 19 days. After cleaning the rooms thoroughly with disinfectant $\left(\right.$ Rely $^{+}$On Virkon, LANXESS, or RUBYSTA in Japan), we tested 15 areas that were in close contact with the patient and medical staff. Swabs were used to transfer 5 environmental samples from room A and 10 samples from room B to universal transport media (Copan, Murrieta, CA). Cleaning was conducted immediately after the patient left the rooms. Environmental sampling was conducted within 5 days and $30 \mathrm{~min}$ after the patient left rooms $\mathrm{A}$ and $\mathrm{B}$, respectively. Nucleic acids were extracted using MagMAX Viral/Pathogen Nucleic Acid Isolation Kit (ThermoFisher Scientific, Waltham, $\mathrm{MA}$ ) and were tested using real-time reverse transcription polymerase chain reaction (RT-PCR) targeting the nucleocapsid $(N)$ gene of SARS-CoV-2. Seven sets of primers and probes (CDC-N1, CDC-N2, CDC-N3, YCH-N1, YCH-N2, NIID-N1, and NIID-N2) were used to detect SARS-CoV-2 as previously described (Supplemental Table 1 online). ${ }^{3}$ For the internal positive control, the human ribonuclease $\mathrm{P} 30$ subunit ( $R P P 30$ ) gene was used. The patient's records, timing of cleaning and sampling, and RT-PCR results were collated.

Author for correspondence: Yosuke Hirotsu, E-mail: hirotsu-bdyu@ych.pref. yamanashi.jp

Cite this article: Hirotsu Y, et al. (2020). Environmental cleaning is effective for the eradication of severe acute respiratory syndrome coronavirus 2 (SARS-CoV-2) virus in contaminated hospital rooms: A patient from the Diamond Princess cruise ship. Infection Control \& Hospital Epidemiology, 41: 1105-1106, https://doi.org/10.1017/ ice.2020.144
On admission, the patient had fever $\left(39^{\circ} \mathrm{C}\right)$ and a mild cough (Supplemental Table 2 online). The chest X-ray and computed tomography scan on day 1 showed signs of pneumonia in both lungs. He received lopinavir/ritonavir and antibacterial therapy on day 2, but showed respiratory failure. He received supplemental oxygen from day 4 to day 15 . After careful clinical management, the patient's overall status improved. RT-PCR showed that his sputum was positive for SARS-CoV-2 on day 11. Subsequently, nasopharyngeal swabs were negative on days 17, 22, and 29.

The patient stayed in room A for 3 days, during which he had the SARS-CoV-2 infection. After cleaning room A, 5 environmental samples were examined by RT-PCR. All samples were negative for SARS-CoV-2 and were positive or negative for RPP30 (Table 1).

After the patient left room $A$, he resided in room $B$ for 20 days. Ten environmental samples were collected after cleaning. All 10 samples from room $B$ were negative for SARS-CoV-2 and were positive or negative for RPP30 (Table 1).

SARS-CoV-2 is detectable in several types of clinical samples including bronchial lavage fluid, nasopharyngeal swab, pharyngeal swab, sputum, saliva, and feces. ${ }^{4,5}$ Transmission of SARS-CoV-2 via surfaces in hospitals is of great concern to medical staff and patients. Blocking the potential routes of transmission is essential for preventing the spread of SARS-CoV- $2 .{ }^{6}$ A recent study showed that environmental contamination can occur via contact with patients with SARS-CoV-2 and upper respiratory tract symptoms. ${ }^{7}$ After cleaning, all areas were negative for SARS-CoV-2; therefore, thorough cleaning is sufficient for SARS-CoV-2 decontamination.

This study had several limitations. First, RT-PCR was not performed before cleaning because of the risk of nosocomial transmission. Therefore, a comparison of the viral loads of high-touch areas before and after cleaning is required. Second, this study involved a single patient, and further studies are required to confirm these findings.

In summary, our data indicate the effectiveness of environmental cleaning for SARS-CoV-2 decontamination. This information is useful for infection control strategies and may alleviate the concerns of medical staff. 
Table 1. Real-Time RT-PCR Analysis of Environmental Samples

\begin{tabular}{|lll}
\hline Location & \multicolumn{1}{c}{ RT-PCR (No. of Samples) } \\
\hline Patient room A & SARS-CoV-2 & Human RPP30 \\
\hline Light switch & Negative $(0 / 1)$ & Negative $(0 / 1)$ \\
\hline Nurse call attached to the bed & Negative $(0 / 1)$ & Positive $(1 / 1)$ \\
\hline Toilet door handle & Negative $(0 / 1)$ & Negative $(0 / 1)$ \\
\hline Bed guard & Negative $(0 / 1)$ & Positive $(1 / 1)$ \\
\hline Anterior room A & & \\
\hline Dust box & Negative $(0 / 1)$ & Positive $(1 / 1)$ \\
\hline Patient room B & & \\
\hline Bed desk & Negative $(0 / 1)$ & Negative $(0 / 1)$ \\
\hline Bed guard & Negative $(0 / 1)$ & Negative $(0 / 1)$ \\
\hline Door handle & Negative $(0 / 1)$ & Positive $(1 / 1)$ \\
\hline Dust box, room side & Negative $(0 / 1)$ & Negative $(0 / 1)$ \\
\hline Dust box, corridor & Negative $(0 / 1)$ & Positive $(1 / 1)$ \\
\hline Control panel on mechanical \\
ventilation & Negative $(0 / 1)$ & Positive $(1 / 1)$ \\
\hline Light switch & & \\
\hline Nurse call & Negative $(0 / 1)$ & Negative $(0 / 1)$ \\
\hline Hand soap dispenser & Negative $(0 / 1)$ & Positive $(1 / 1)$ \\
\hline Anterior room B & Negative $(0 / 1)$ & Negative $(0 / 1)$ \\
\hline Sink, external rim and & & Positive $(1 / 1)$ \\
\hline internal bowl & Negative $(0 / 1)$ & \\
\hline
\end{tabular}

Note. PCR, polymerase chain reaction; RPP30, ribonuclease P 30 subunit; SARS-CoV-2, severe acute respiratory syndrome coronavirus 2 .
Acknowledgments. We thank all of the medical and ancillary hospital staff and the patients for consenting to participate. We thank Suzanne Leech, $\mathrm{PhD}$, from the Edanz Group for editing a draft of this manuscript.

Financial support. This study was supported by a Grant-in-Aid for the Genome Research Project from Yamanashi Prefecture (to M.O. and Y.H.), the Japan Society for the Promotion of Science (JSPS) KAKENHI EarlyCareer Scientists (grant no. JP18K16292 to Y.H.), a Research Grant for Young Scholars (to Y.H.), the YASUDA Medical Foundation (to Y.H.), the Uehara Memorial Foundation (to Y.H.), and Medical Research Grants from the Takeda Science Foundation (to Y.H.).

Conflicts of interest. All authors report no conflicts of interest relevant to this article.

\section{References}

1. Wang D, Hu B, Hu C, et al. Clinical characteristics of 138 hospitalized patients with 2019 novel coronavirus-infected pneumonia in Wuhan, China. JAMA 2020;323:1061-1069.

2. van Doremalen N, Bushmaker T, Morris D, et al. Aerosol and surface stability of SARS-CoV-2 as compared with SARS-CoV-1. N Engl J Med 2020;382: 1564-1567.

3. Hirotsu Y, Mochizuki H, Omata M. Double-quencher probes improved the detection sensitivity of severe acute respiratory syndrome coronavirus 2 (SARS-CoV-2) by one-step RT-PCR. medRxiv 2020. doi: 10.1101/2020.03. 17.20037903.

4. Wang W, Xu Y, Gao R, Lu R, Han K, Wu G, Tan W. Detection of SARS-CoV-2 in different types of clinical specimens. JAMA 2020. doi: 10.1001/jama.2020.3786.

5. To KK, Tsang OT, Chik-Yan Yip C, et al. Consistent detection of 2019 novel coronavirus in saliva. Clin Infect Dis 2020. doi: 10.1093/cid/ ciaal49.

6. Kampf G, Todt D, Pfaender S, Steinmann E. Persistence of coronaviruses on inanimate surfaces and their inactivation with biocidal agents. J Hosp Infect 2020;104:246-251.

7. Ong SWX, Tan YK, Chia PY, et al. Air, Surface environmental, and personal protective equipment contamination by severe acute respiratory syndrome coronavirus 2 (SARS-CoV-2) from a symptomatic patient. JAMA 2020. doi: 10.1001/jama.2020.3227.

Supplementary material. To view supplementary material for this article please visit https://doi.org/10.1017/ice.2020.144

\section{COVID-19 affects healthy pediatricians more than pediatric patients}

\section{Nima Rezaei MD, PhD (1)}

Research Center for Immunodeficiencies, Children's Medical Center, Tehran University of Medical Sciences, Tehran, Iran, Department of Immunology, School of Medicine, Tehran University of Medical Sciences, Tehran, Iran and Network of Immunity in Infection, Malignancy and Autoimmunity (NIIMA), Universal Scientific Education and Research Network (USERN), Tehran, Iran

To the Editor-Coronavirus disease 2019 (COVID-19) emerged in China in late December and has spread rapidly throughout the world. The World Health Organization (WHO) considers it a pandemic. In $\sim 4$ months from start of this outbreak, as of April 4, 2020, $>1,000,000$ patients had been affected. ${ }^{1}$

Meanwhile, children appear less likely to be affected than adults; among the affected children, most have mild symptoms and some are even asymptomatic. ${ }^{2-4}$ The Chinese Center for

Author for correspondence: Nima Rezaei, E-mail: rezaei_nima@tums.ac.ir Cite this article: Rezaei N. (2020). COVID-19 affects healthy pediatricians more than pediatric patients. Infection Control \& Hospital Epidemiology, 41: 1106-1107, https:// doi.org/10.1017/ice.2020.139
Disease Control and Prevention has reported that among $\sim 70,000$ cases, $<1 \%$ were aged $<10$ years. ${ }^{5}$

The first official COVID-19 case in Iran was announced on February 19, 2020. On March 16, 2020, 4 weeks later, the daily situation report on COVID-19 showed that $<1 \%$ of $\sim 5,000$ confirmed cases were children aged $<10$ years. ${ }^{6}$ The Children's Medical Center in Iran has 348 beds for pediatric patients, with bed occupancy rate of $94.7 \%$ and an average length of stay of 3.3 days. During the 4 -week period after the first identified case in the country, only 3 positive COVID-19 cases were identified, for an estimated frequency of 1 per 1,000 admitted pediatric patients. Meanwhile, among 60 pediatricians working in this hospital, 5 were positive for COVID-19 by real-time reverse 